\title{
Dispositivos nanofotónicos: enfocar y concentrar la luz a escalas nanométricas
}

\author{
Rafael Salas-Montiel* y Sylvain Blaize*
}

RESUMEN: El confinamiento de señales ópticas a escalas nanométricas y el aumento de su intensidad óptica como consecuencia de ese confinamiento representa un avance para el desarrollo de sensores ópticos y de nuevas fuentes eficientes y ultracompactos en aplicaciones como la transmisión de la información cuántica y la biomedicina. Sin embargo, estos efectos ópticos son inalcanzables con dispositivos fabricados con materiales dieléctricos debido a que éstos están limitados por la difracción. El uso de materiales metálicos con estructuras de dimensiones nanométricas que soportan ondas ópticas de superficie llamadas plasmones-polaritones de superficie permite sobrepasar este límite de difracción y confinar la luz a escalas nanométricas. En este trabajo, publicado en la revista Nano Letters, se presenta el confinamiento de luz en nanoestructuras plasmónicas integradas sobre guías de ondas de silicio. Se reveló un confinamiento extremo de la luz por medio de la microscopía óptica de barrido en campo cercano. Los resultados de este trabajo abren nuevas perspectivas para aplicaciones en la información cuántica, las telecomunicaciones y la biomedicina.

PALABRAS CLAVE: plasmones-polaritones de superficie, plasmónica integrada, microscopía óptica de barrido en campo cercano, nanofotónica, fotónica en silicio.

ABSTRACT: The confinement and the enhancement of light into the nanometer scale represent an advance in the development of efficient and compact optical sources and sensors, useful in quantum information and biomedical applications. However, the optical confinement of light to the nanometer scale cannot be reach with optical components fabricated with dielectric materials because of the diffraction limit. The use surface plasmons-polaritons supported by metallic nanostructures overcomes this limit and therefore, confines the light to the subwavelength scale. In this contribution, recently published in Nano Letters, we demonstrated the confinement and the enhancement of light in integrated plasmonic structures on silicon photonics. With the use of near field scanning optical microscopy, we reveled the extreme confinement of light. This phenomenon opens novel routes for the development of quantum information, telecommunications, and biomedical applications.

KEYWORDS: surface plasmon-polaritons, plasmonics, integrated plasmonics, near field scanning optical microscopy, nanophotonics, silicon photonics.

La nanofotónica es una rama de la fotónica que estudia los fenómenos de interacción entre la luz y la materia a escalas nanométricas (Gaponenko, 2010). Esta luz, generada por láseres, interactúa con estructuras que presentan

Recibido: 20 de noviembre de 2015. Aceptado: 25 de noviembre de 2015.

* Laboratoire de Nanotechnologie et d'Instrumentation Optique, Institut Charles Delaunay, CNRS UMR 6281, Université de technologie de Troyes, 12 rue Marie Curie, 10004 Troyes, France. Correspondencia: (rafael.salas@utt.fr). Teléfono: +33(0)3 25718001. 
dimensiones inferiores a la longitud de onda de los láseres para producir efectos ópticos de interés. Estos fenómenos son generalmente producidos en la superficie de nanoestructuras, de tal manera que se pueden controlar o reforzar con tan solo modificar el tamaño, la forma o los materiales de éstas. Un efecto óptico de interés en el campo de la nanofotónica es el de focalizar y concentrar un haz de luz láser en volúmenes a escalas nanométricas (Gramotnev et al., 2014). El fenómeno de focalización es bien conocido en óptica geométrica y ocurre cuando los rayos de luz provenientes de un láser colimado convergen en un punto único llamado foco al atravesar una lente. Sin embargo, el haz de luz láser en este punto focal presenta un volumen limitado por las leyes de difracción y por lo tanto superior a $(\lambda / 2 n)^{3}$, donde $\lambda$ es la longitud de onda de la luz láser y $n$ el índice de refracción del medio por el que se propaga. La nanofocalización permite enfocar el haz de luz láser a un volumen inferior al impuesto por la leyes de difracción. Este fenómeno de concentración a escalas nanométricas refuerza la intensidad de la luz láser lo que permite una interacción entre la luz y la materia a niveles que alcanzan el fotón único o la molécula única en circuitos fotónicos integrados. El enfoque de la luz a escalas nanométricas, por ejemplo, encuentra aplicaciones en el desarrollo de pinzas ópticas para la biología o la medicina (Ashkin et al., 1987). Estas pinzas ópticas utilizan un haz de luz láser enfocado para sujetar o seleccionar objetos microscópicos o nanoscópicos tales como moléculas individuales (baterías, virus, células o incluso ramas de ADN). El calor local generado por el enfoque de la luz también puede ser utilizado en bionanotecnología para el control de la hibridación del ADN (Hamad-Schifferli et al., 2002). Por otro lado, la amplificación de la interacción entre la luz y la materia encuentra aplicaciones en óptica no lineal para reforzar efectos como la generación del segundo armónico y en óptica cuántica para exaltar las propiedades de emisión de emisores cuánticos. Hoy en día, muchos centros e institutos de investigación trabajan en nanofotónica para la realización de nanodispositivos ópticos y optoelectrónicos integrados.

En este contexto, en colaboración con el grupo del profesor Ali Adibi del Instituto de Tecnología de Georgia, en Atlanta, Estados Unidos, desarrollamos, fabricamos y estudiamos nanoestructuras metálicas colocadas sobre guías ondas de silicio para alcanzar un confinamiento extremo y una exaltación de la luz (Luo et al., 2015). Esta estructura metálica soporta excitaciones electromagnéticas llamadas plasmones-polaritones de superficie (PPS) que se propagan a lo largo de ella y que permiten este fenómeno óptico de nanofocalización al reducir gradualmente sus dimensiones laterales. En su forma más simple, un PPS es una onda electromagnética de superficie que se propaga a lo largo de superficies o películas delgadas metálicas y cuya amplitud decae exponencialmente en puntos alejados de estas superficies (Raether, 1988). Una de las características principales de los PPS es la de poseer un vector de onda $\mathbf{k}$ de magnitud superior al de las ondas electromagnéticas en el aire para una misma frecuencia $\omega$. Esto implica que los PPS no pueden ser 
FIGURA 1. Dispositivo nanofotónico para la focalización de la luz. a) Esquema tridimensional del nanodispositivo para el enfoque y la concentración de la luz. b) Imagen realizada por medio de microscopía electrónica de barrido de la estructura triangular de oro depositada sobra una guía de ondas óptica de silicio.

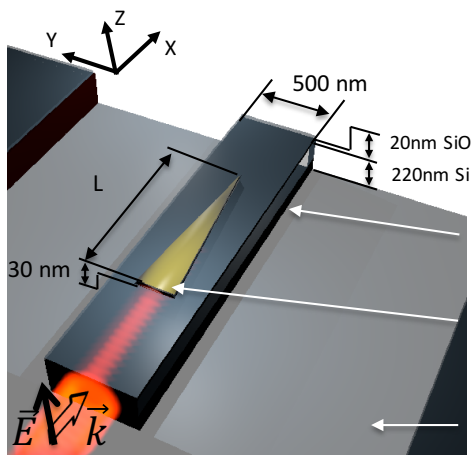

(a)

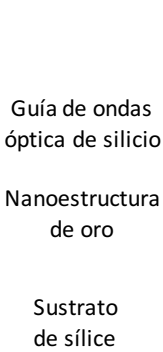

de sílice

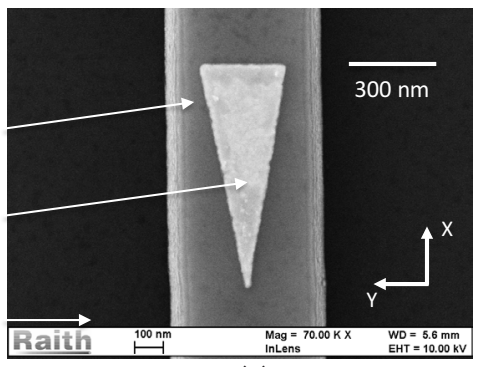

(b)

Fuente: Elaboración propia.

excitados directamente por ondas electromagnéticas viajando en el aire sino que necesitan estructuras ópticas superficiales que aumenten la magnitud del vector de onda de la luz para empatar la magnitud del vector de onda de los PPS. Es decir, la energía y el momentum deben conservarse. Entre las estructuras superficiales conocidas y utilizadas para excitar PPS encontramos los prismas (Otto, 1968; Kretschmann, 1971) y las rejillas de difracción (De la Cruz et al., 2012). Existen otro tipo de estructuras altamente eficientes para excitar PPS como las guías de ondas ópticas. Efectivamente, estas estructuras ópticas soportan modos fotónicos que presentan magnitudes de su vector de onda equivalentes al de los PPS y, por lo tanto, éstos pueden ser excitados eficazmente. Es precisamente con el modo fotónico de una guía de ondas en silicio que se logró excitar a los PPS de la nanoestructura metálica y obtener la focalización de la luz a escalas nanométricas.

Este dispositivo nanofotónico consiste por lo tanto de una guía de onda óptica de silicio sobre la cual se colocó un nanotriángulo isósceles de oro (figura 1a).

Para excitar la guía de ondas óptica de silicio se utilizó una fibra óptica de sílice microlente conectada a una fuente de luz láser con una longitud de onda de 1,550 nm y con una polarización lineal. Esta fuente permite excitar el modo fundamental transversal magnético de la guía de ondas de silicio. La nanoestructura metálica se excita, a su vez, por medio de este modo fundamental transversal magnético que se propaga a lo largo de la guía de ondas óptica de silicio (figura 1a). Este modo se acopla eficazmente con los modos híbridos plasmónicos-fotónicos soportados por la nanoestructura metálica. Conforme estos modos híbridos se propagan a lo largo de la nanoestructura se transforman gradualmente en modos plasmónicos. De tal manera que al final de la estructura, en la punta del nanotriángulo, el modo es puramente 
FIGURA 2. Imagen experimental obtenida por microscopía óptica de barrido en campo cercano del nanodispositivo fotónico. a) Topografía de la nanoestructura. b) Imagen óptica en campo cercano de la propagación de modos ópticos a lo largo del circuito fotónico integrado que demuestra el confinamiento de la luz a escalas nanométricas y su refuerzo como consecuencia de ese confinamiento.

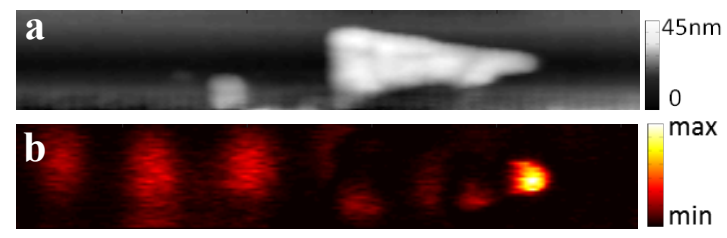

\section{$300 \mathrm{~nm}$}

Fuente: Elaboración propia.

plasmónico. El plasmón-polaritón de superficie queda confinado en la punta del triángulo en volúmenes nanométricos, y este confinamiento genera un refuerzo de la amplitud del campo electromagnético en ese punto con respecto a la amplitud del campo óptico incidente. La transformación del modo fotónico al plasmónico se hace con muy pocas pérdidas de energía electromagnética.

Para fabricar esta nanoestructura, se utilizó un proceso de litografía electrónica. Esta técnica consiste en hacer un barrido con un haz de electrones enfocado sobre la superficie de un sustrato recubierto por una resina de polímero sensible a los electrones. Para trazar el nanotriángulo, el haz de electrones es controlado por medio de un software de manera precisa para exponer sólo la región triangular. Una vez expuesto, la resina es revelada mediante un revelador químico que elimina la parte expuesta de la resina de tal manera que en la resina queda un hoyo de forma triangular. Finalmente, se deposita una película de oro de $30 \mathrm{~nm}$ de espesor sobre esta superficie por medio de una técnica de evaporación térmica. La película delgada de oro se deposita sobre la guía de silicio (pasando a través de la región hueca de la resina) y sobre la resina en la parte no expuesta. El excedente de oro sobre la resina se retira al sumergir la muestra en solventes que disuelve la resina no expuesta. Este proceso de retiro del oro sobre la resina no expuesta al haz de electrones se le conoce con el término en inglés de lift-off.

Las propiedades ópticas del nanodispositivo diseñado y fabricado se verificaron experimentalmente con microscopía óptica de barrido en campo cercano (NSOM, por sus siglas en inglés). A diferencia de la microscopía óptica común cuya resolución espacial está acotada por el límite de difracción $(\lambda / 2 n)$, la microscopía óptica de barrido en campo cercano permite sondear el campo eléctrico local en la vecindad de la nanoestructura con una resolución espacial de algunos nanómetros, muy por debajo del límite de difracción que se encuentra en un microscopio óptico común. Por lo tanto, es una técnica especialmente bien adaptada para la medición y observación de plasmones-polaritones de superficie (Novotny, 2009). 
Podemos observar tanto la topografía de la superficie de la nanoestructura (figura 2a) como la intensidad superficial de la propagación del PPS a lo largo de ésta (figura 2b).

En la figura $2 \mathrm{~b}$ se distingue la propagación del modo fotónico a lo largo de la guía de ondas de silicio, justo antes de llevar a la base de la nanoestructura. De igual forma, se observa la luz focalizada y confinada en un volumen a escalas nanométricas, muy por debajo del límite de difracción. Finalmente, se observa el incremento de la intensidad del campo eléctrico en la punta de la nanoestructura metálica con respecto a la intensidad del campo eléctrico incidente.

Este trabajo reveló las propiedades de confinamiento de la luz a escalas nanométricas y el aumento de la intensidad luminosa en nanodispositivos plasmónicos integrados basados en silicio. El uso de métodos de nanofabricación y el desarrollo de métodos de caracterización adaptados a la observación de plasmones-polaritones de superficie permitió obtener estos resultados. El confinamiento y el refuerzo de la luz en escalas nanométricas abre nuevas perspectivas para el desarrollo de aplicaciones tales como nanopinzas ópticas, nanosensores moleculares o para el desarrollo de fuentes eficientes de fotones individuales.

\section{Bibliografia}

Ashkin A., Dziedzic J.M. y Yamane T. (1987). Optical trapping and manipulation of single cells using infrared laser beams. Nature, vol. 330: 769.

De la Cruz S., Méndez E. R., Macías D., Salas-Montiel R., y Adam P. M. (2012). Compact surface structures for the efficient excitation of surface plasmon-polaritons. Physica Status Solidi B, vol. 249, núm. 6: 1178.

Gaponenko, S. V. (2010). Introduction to nanophotonics. Cambridge University Press.

Gramotnev, D. K. y Bozhevolnyi, S. I. (2014). Nanofocusing of electromagnetic radiation. Nature Photonics, vol. 8, núm. 1: 13.

Hamad-Schifferli K., Schwartz, J. J., Santos A.T., Zhang S., y Jacobson J. M. (2002). Remote electronic control of DNA hybridization through inductive coupling to an attached metal nanocrystal antenna. Nature, vol. 415: 152.

Kretschmann, E. (1971). Die Bestimmung optischer Konstanten von Metallen durch Anregung von Oberflächenplasmaschwingungen. Zeitschrift für Physik, vol. 241: 313.

Luo, Y., Chamanzar, M., Apuzzo A., Salas-Montiel R., Nguyen K.N., Blaize S., y Adibi A. (2015). On-chip hybrid photonic-plasmonic light concentrator for nanofocusing in an integrated silicon photonics platform. Nano Letters, vol. 15, núm. 2: 849. Novotny, L. y Hecht, B. (2009). Principles of Nano-optics. Cambridge University Press.

Otto, A. (1968). Excitation of nonradiative surface plasmon waves in silver by the method of frustrated total reflection. Zritschrift für Physik, vol. 216: 398.

Raether, H. (1988). Surface plasmons on smooth and rough surfaces and on gratings, Springer Tracts in Modern Physics, vol. 111. Springer-Verlag. 